\title{
Metabolic reprogramming by inhibition of prolyl hydroxylases protects alveolar epithelial cells from LPS-neutrophil-induced energy derangements and cell death
}

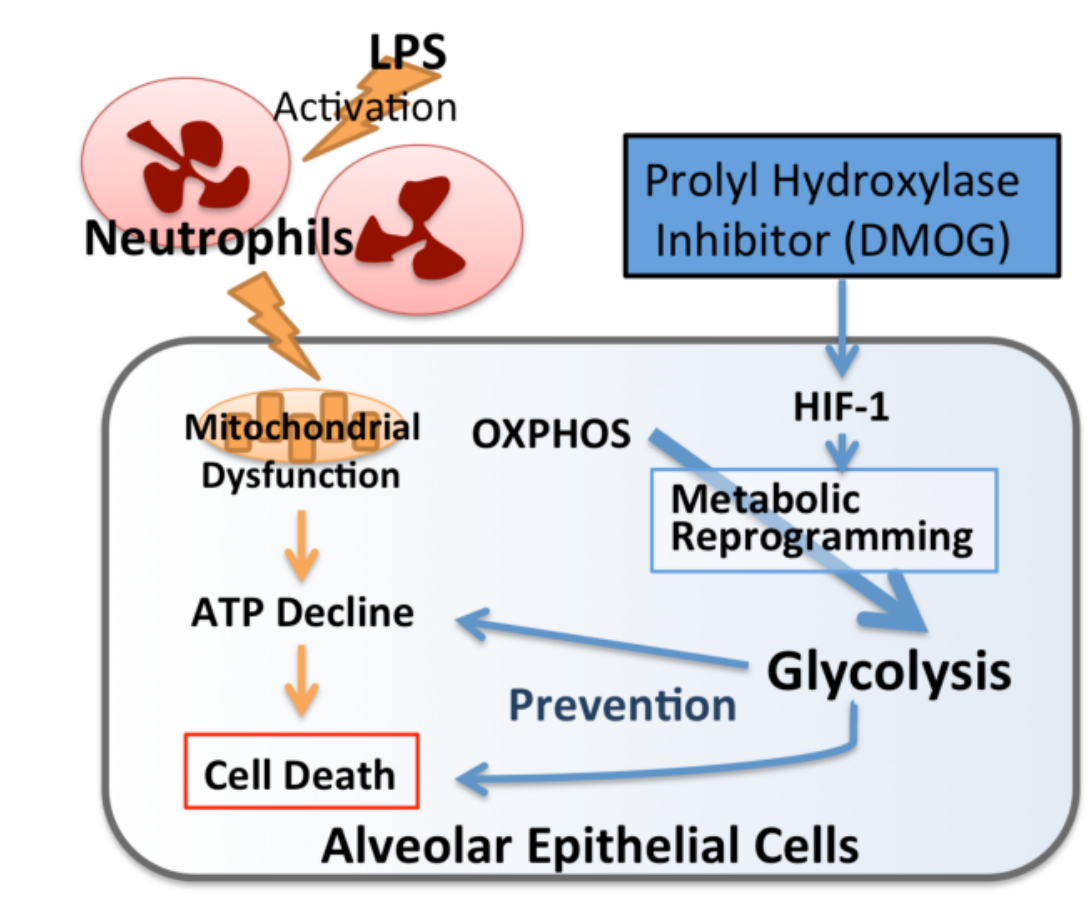

\section{Background}

* Neutrophil-mediated alveolar epithelial injury is a hallmark of acute respiratory distress syndrome (ARDS).

* Cellular energy derangement due to mitochondrial dysfunction underlies the alveolar epithelial injury.

* Therefore, metabolic reprogramming shifting bioenergetic activity from mitochondria-dependent OXPHOS to glycolysis may protect alveolar epithelial cells from ARDS.

\section{Hypothesis}

Metabolic reprogramming by pharmacological inhibition of a cellular oxygen sensor, prolyl hydroxylases (PHDs) may protect alveolar epithelial cells from lung injury.
Kentaro Tojo, Nao Tamada, Yusuke Nagamine, Shuhei Ota, Takahisa Goto

Yokohama City University Graduate School of Medicine, Dept of Anaesthesiology \& Intensive Care, Yokohama, Japan

\section{Summary}

- LPS-activated neutrophils cause ATP decline and alveolar epithelial cell death.

- Pharmacological inhibition of a cellular oxygen sensor, prolyl hydroxylases (PHDs) causes HIF-1 mediated metabolic reprogramming from oxidative phosphorylation (OXPHOS) to glycolysis.

- PHD inhibition protected alveolar epithelial cells from neutrophil-LPS-induced ATP decline and cell death via HIF-1-mediated metabolic reprogramming.

\section{Results and Discussion}

Neutrophils + LPS induces ATP-decline and alveolar epithelial cell death
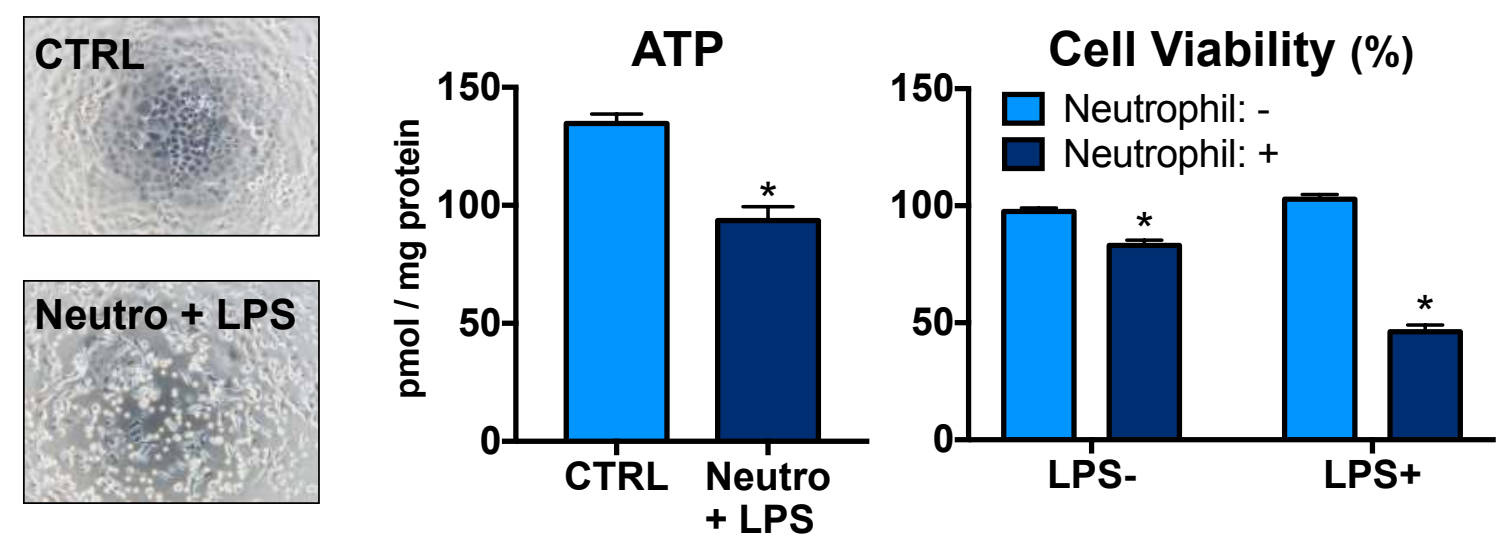

Neutrophil-LPS challenges induced ATP decline in MLE12 cells; an alveolar epithelial cell line Concomitantly, cell viability was significantly decreased by neutrophil-LPS challenge. The cell death was not suppressed by caspase $3 / 7$ inhibitor (data not shown). ${ }^{*} p<0.05 \mathrm{vs}$. control.
Methods

Cell culture experiments was added to the culture medium

In glycolytic inhibition experiments, 2-DG (2-deoxy-D-glucose: a glycolytic inhibitor)

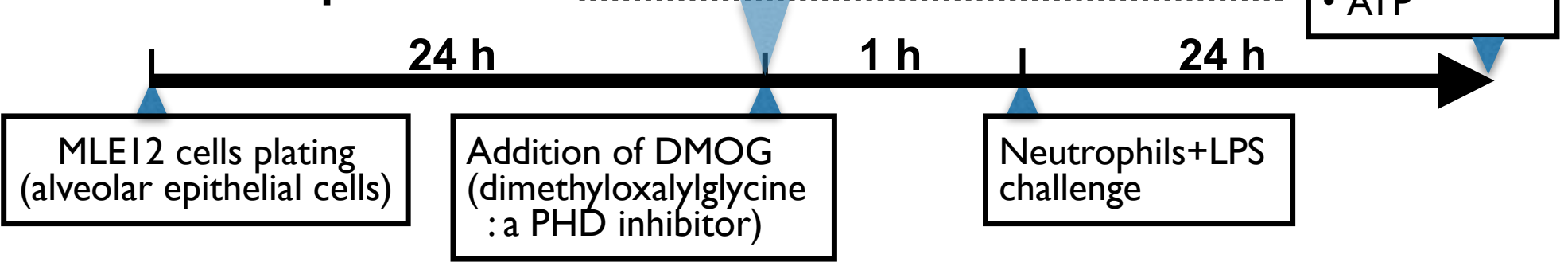

Animal experiments

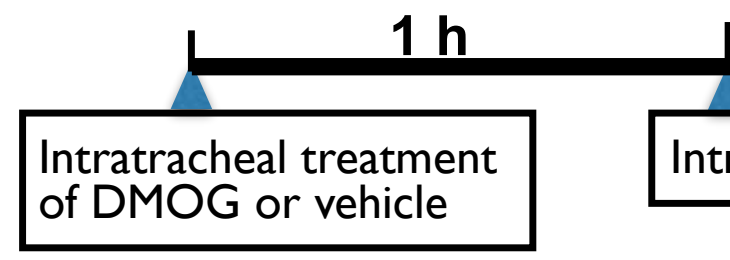

$15 h$

Euthanization

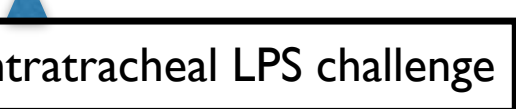

Analysis of

- Cytokeratin 18 in BALF :an epithelial cell death marker

DMOG protects alveolar epithelial cell through HIF-I-mediated metabolic reprogramming from OXPHOS to glycolysis.

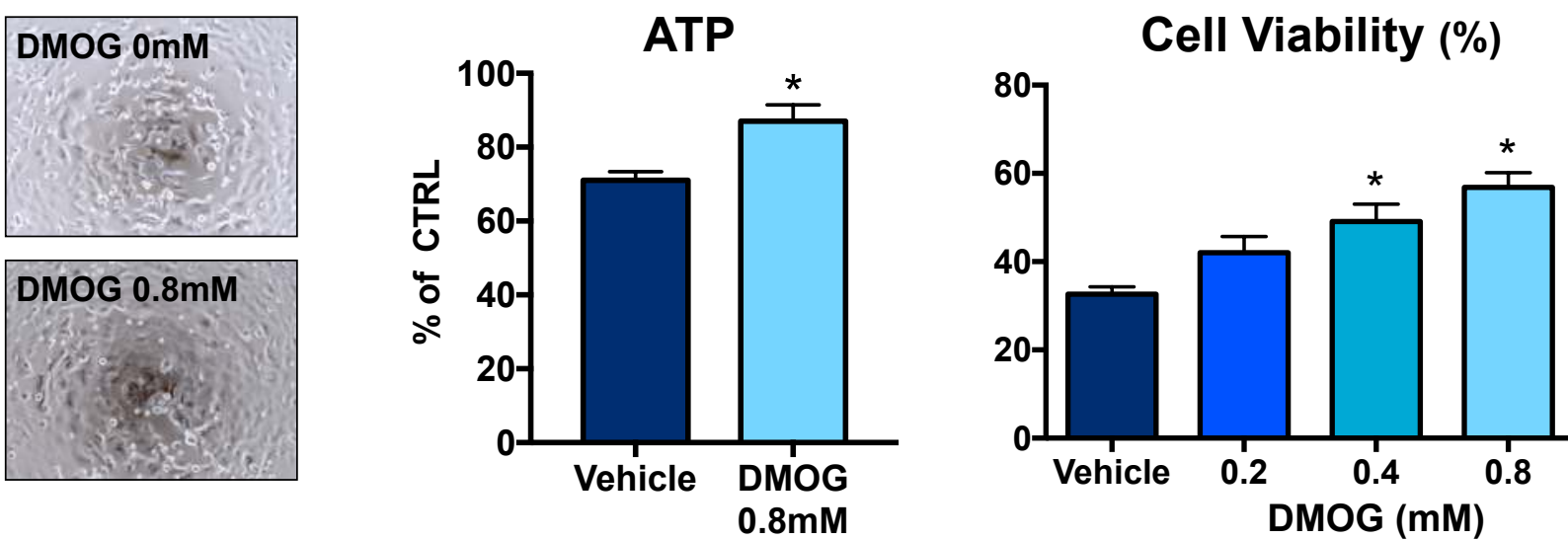

DMOG treatment attenuated the neutrophil-LPS-induced ATP decline and cell death in dose-dependent manner. ${ }^{*} p<0.05$ vs. vehicle group

Intratracheal DMOG treatment attenuates alveolar epithelial cell death and ATP decline in LPS-induced lung injury mice

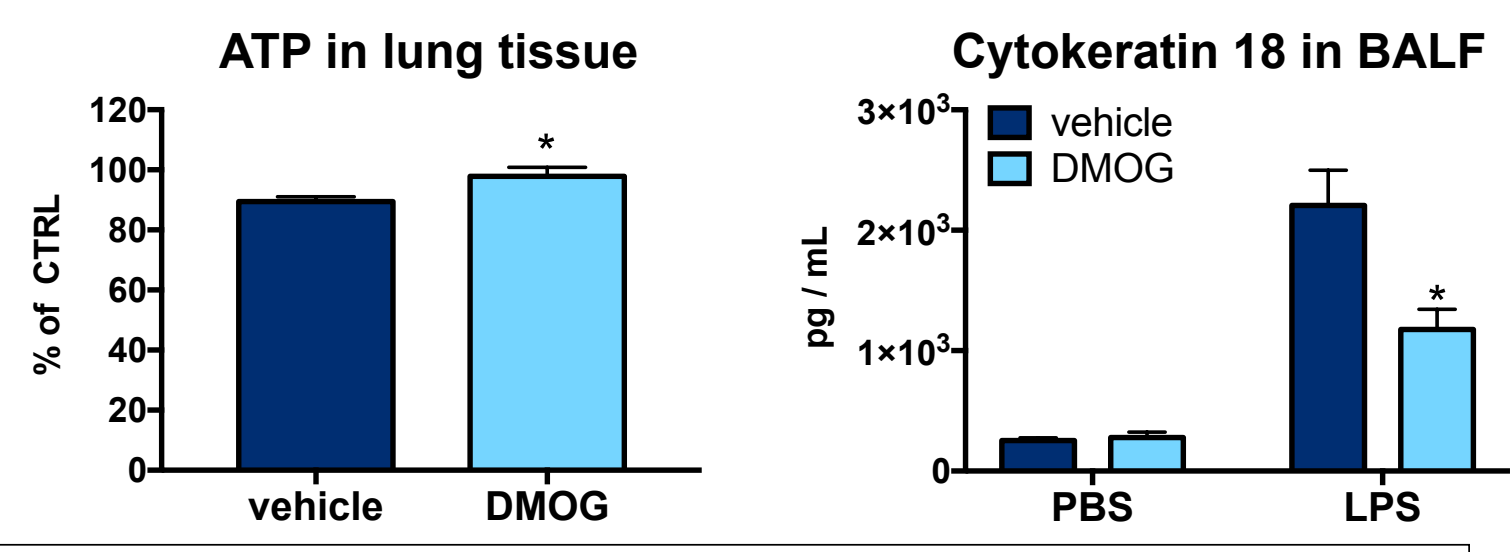

Intratracheal LPS-instillation-induced ATP decline in lung tissues and increased an epithelial cell death marker; cytokeratin 18 in BALF. Intratracheal DMOG treatment attenuated these changes. $\mathrm{p}<0.05$ vs. vehicle group.
Pharmacological inhibition of PHDs by DMOG causes HIF-I mediated metabolic reprogramming

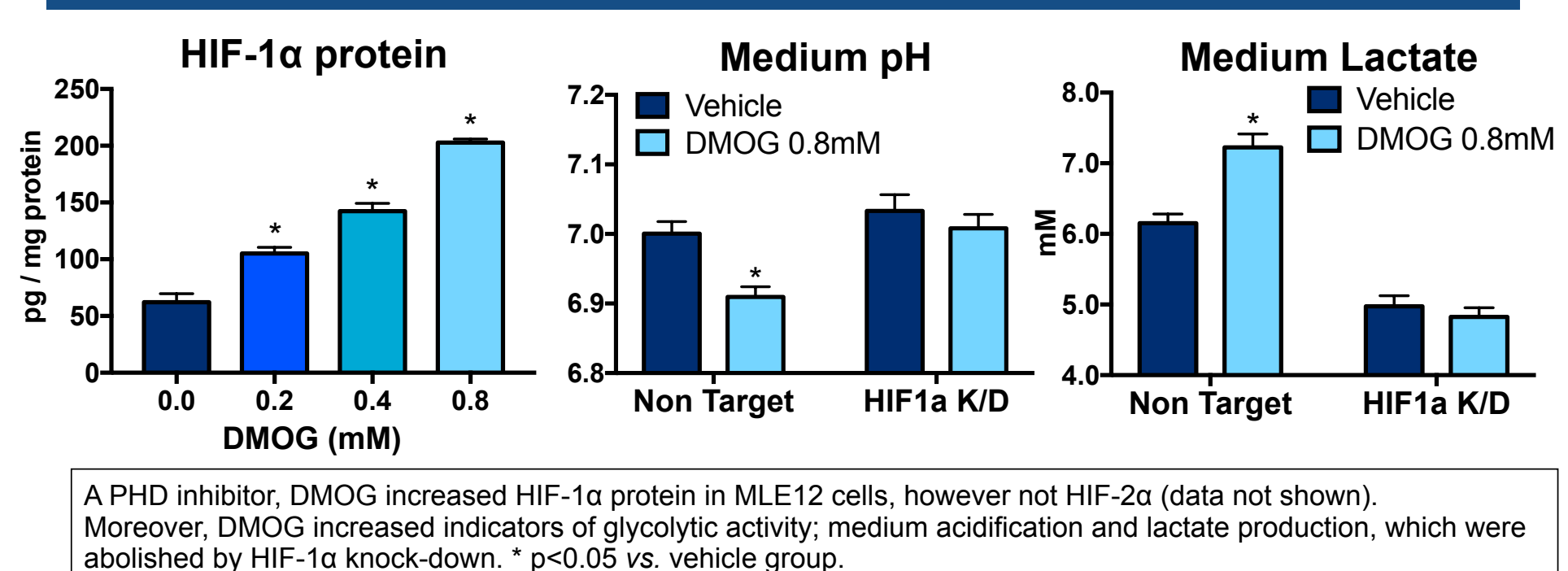
abolished by HIF-1a knock-down. " $p<0.05$ vs, vehicle group.
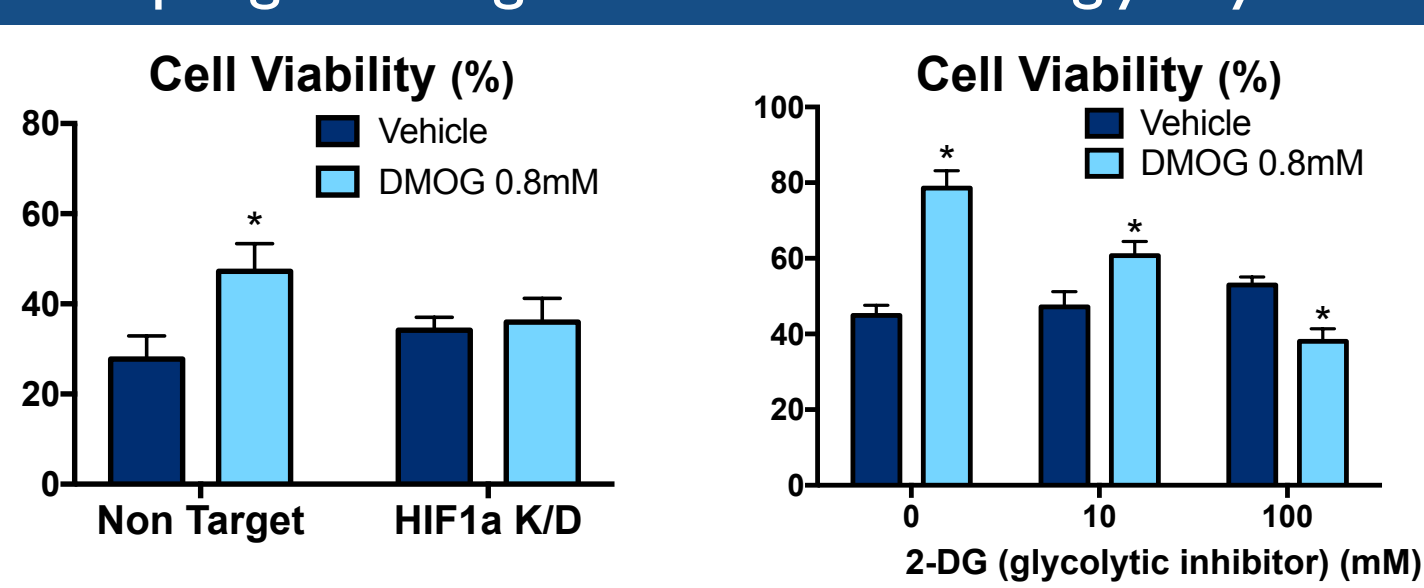

HIF-1a knock-down abolished the protective effects of DMOG. Furthermore, glycolytic inhibition by 2-DG reversed the effects of DMOG. * $\mathrm{p}<0.05$ vs. vehicle group

\section{Conclusion}

- Pharmacological inhibition of PHDs protected alveolar epithelial cells from LPS-neutrophilinduced ATP decline and cell death via HIF-1mediated metabolic reprogramming.

- Metabolic reprogramming from OXPHOS to glycolysis may be a novel approach to protect alveolar epithelial cells from ARDS. 